\title{
Epithelioid sarcoma of the spine: a case report and review
}

\author{
Jeong Hyun Yoo ${ }^{1 *}$, Sun Hee Sung ${ }^{2}$ \\ ${ }^{1}$ Department of Radiology, Mokdong Hospital, Ewha Womans University School of Medicine, Seoul, Korea; \\ ${ }^{2}$ Department of Pathology, Mokdong Hospital, Ewha Womans University School of Medicine, Seoul, Korea. \\ Email: yoolee@ewha.ac.kr
}

Received 20 April 2011; revised 24 May 2011; accepted 8 June 2011.

\begin{abstract}
Epithelioid sarcoma is a rare malignant soft tissue neoplasm commonly involving extremities of young adults and rarely occurring in the trunk area. It resembles a chronic inflammatory process and mimics benign reactive granuloma or other benign diseases. Despite its indolence and slow growth, the prognosis is poor with high recurrence and early lymph node spreading and hematogeneous distant metastasis. Involvement of the spine is extremely rare and difficult to diagnose correctly in clinical presentation and pathology. We describe our experience a case of epithelioid sarcoma involving lumbar spine. A pre-operative spine MRI showed a large solid and necrotic paraspinal mass which extended into the spinal canal with cord compression, and bone destruction. Final correct diagnosis was confirmed by immunohistochemical studies. When young adults present with a spinal lesion that has unusual large necrotic soft tissue mass, immunohistochemical studies are recommended for promptly determining the possibility of spinal epithelioid sarcoma.
\end{abstract}

Keywords: Sarcoma; Epithelioid: Spine;

Neoplasm: MR Image

\section{INTRODUCTION}

Epithelioid sarcoma is a rare malignant soft tissue neoplasm which occurs most commonly in the extremities in young adults [1-5]. The diagnosis of epithelioid sarcoma has been considered difficult both for clinicians and pathologists because of its relative indolence and slow growth, as well as resemblance to other benign tumors [2-4]. Despite its slow growth, the prognosis of epithelioid sarcoma is poor because it has extreme aggressiveness and has a high tendency for local recurrence and lymphatic or hematogeneous metastasis [1-3,5-8]. Spinal epithelioid sarcoma is extremely rare $[9,10]$ and is very difficult to diagnose correctly [2-4,8-10]. We report a case of epithelioid sarcoma of the lumbar spine with review of literature.

\section{CASE PRESENTATION}

A 29-year-old man presented with left leg weakness which started 5 months earlier. He underwent curettage and posterior fixation of the L1-3 vertebra in a different hospital in response to a misdiagnosis of spondylitis. Initial pathologic diagnosis was inflammatory reaction with fibrosis (not shown). Symptoms did not improve after original treatment. Paralysis and hypoesthesia of the left lower extremity progressed, and a wound infection on his back worsened. The fixated devices were removed with debridement and a tissue biopsy was obtained for the correct diagnosis.

An initial lumbar spine MRI performed at that outside hospital revealed large soft tissue mass involving left L2 vertebral body and paralumbar muscle planes. The mass showed iso- or subtle low signal intensity on the T1-weighted image and heterogeneous slightly high or bright signal intensity on the T2-weighted image. Contrast scan showed diffuse enhancement of the mass with large necrotic non-enhancing portion. The lesion extended into the spinal canal, compressing the spinal cord on the left. Destruction of the lumbar vertebra including left pedicle and transverse process were also present (Figure 1).

The histopathology showed plump and epithelioid appearance of tumor cells, arranged in a granuloma-like fashion around areas of necrosis (X400). On the immunohistochemical stain, the tumor cells showed diffuse strong positive reaction in EMA, as well as in cytokeratin and vimentin. They showed focal positive reaction on CD34. They also showed focal, weakly positive reaction to a smooth muscle actin. Desmin, s-100 protein, CD31, and HMB-45 were all negative. These findings were consistent with epithelioid sarcoma with marked necrosis (Figure 2).

Radical en-block resection of $\mathrm{L} 2$ vertebra and anterior interbody fusion of L1-3 were performed with allogene- 


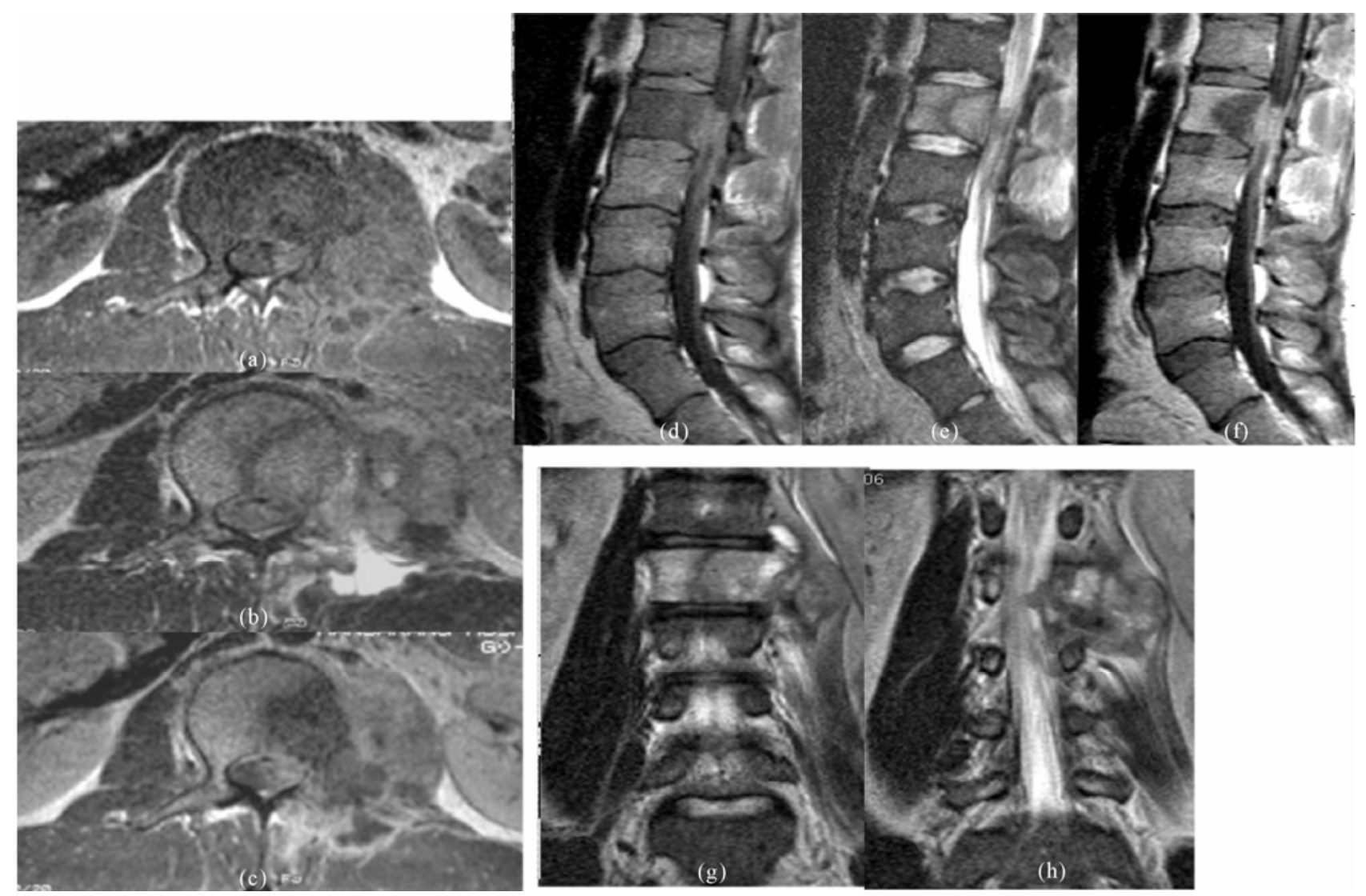

Figure 1. Pre-operative initial MR imaging of lumbar spine. T1-, T2-weighted, and gadolinium enhanced axial (a-c) and sagittal (d-f) scan, and T2-weighted coronal sequential images (g, h). Spine MR images show large soft tissue mass involving left L2 vertebral body and paralumbar muscle planes. The signal intensities of the mass reveal iso- or subtle low signal intensity on the T1-weighted image and heterogeneous slightly high or bright signal intensity on the T2-weighted image. Contrast scans show diffuse enhancement of the mass with large necrotic non-enhancing portion. The lesions extend into the spinal canal compressing spinal cord on the left. Destruction of the lumbar vertebra including left pedicle and transverse process is also noted.

ous bone graft. Radiation and systemic chemotherapy were started. However, the patient's symptoms persisted and follow-up MR showed rapid progression of the disease, with the canal obstruction (not shown). A month later, the patient developed cough and dyspnea. Chest PA and high resolution chest CT showed multifocal hematogeneous metastasis in both lung fields with substantial pleural effusion (not shown). The patient also complained of a left axillary mass, which was consistent with metastasis in the aspiration cytology. The patient refused continued treatment and was discharged against medical advice on patient request.

\section{DISCUSSION}

Spinal involvement of the epithelioid sarcoma is very rare. $[9,10]$. To our knowledge, only two cases of spinal epithelioid sarcoma have been reported. The first report was described in S1 vertebral body and was mistaken for inflammatory disease in clinical and pathologic diagnosis. However, in that case only post-operative image was available without pre-operative radiologic findings. The second case was one in the thoracic spine which showed paraspinal soft tissue mass with spinal canal extension and cord compression, similar to our case except without osseous involvement.

Based on the radiologic findings, large soft tissue mass with necrosis or hemorrhage is characteristic of epithelioid sarcoma. MR signal intensity of the epithelioid sarcoma is mostly isointensity to muscle on T1-weighted image and hyperintensity on T2-weighted image with strong enhancement, Bone invasion is a very rare manifestation of epithelioid sarcoma $[3,7,8]$. If present, it usually consists of localized demineralization or cortical thinning rather than destruction $[1,7,11]$.

Although the second reported thoracic spine case showed paraspinal soft tissue mass with spinal canal extension, it did not show definite tumoral necrosis and bone involvement. MR of our case revealed characteristic large soft tissue mass with necrotic change, although this is very rare in the spine area. Furthermore, our case 


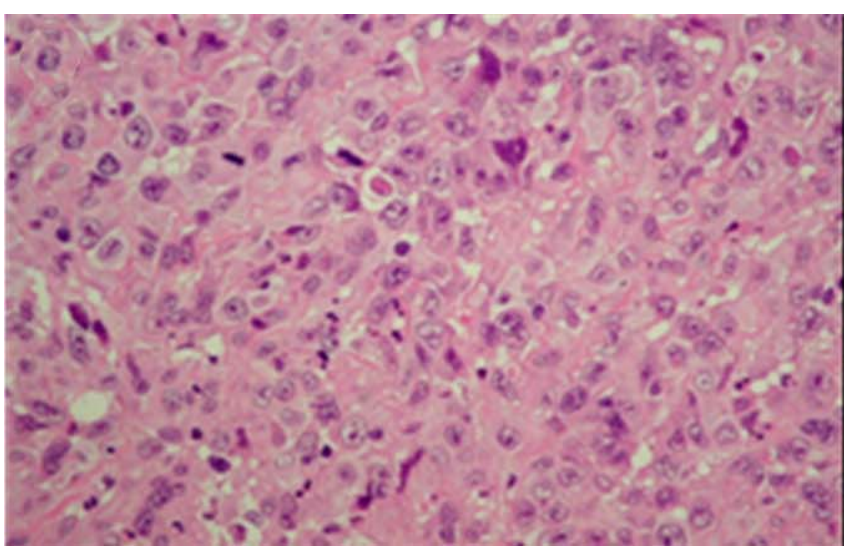

(a)

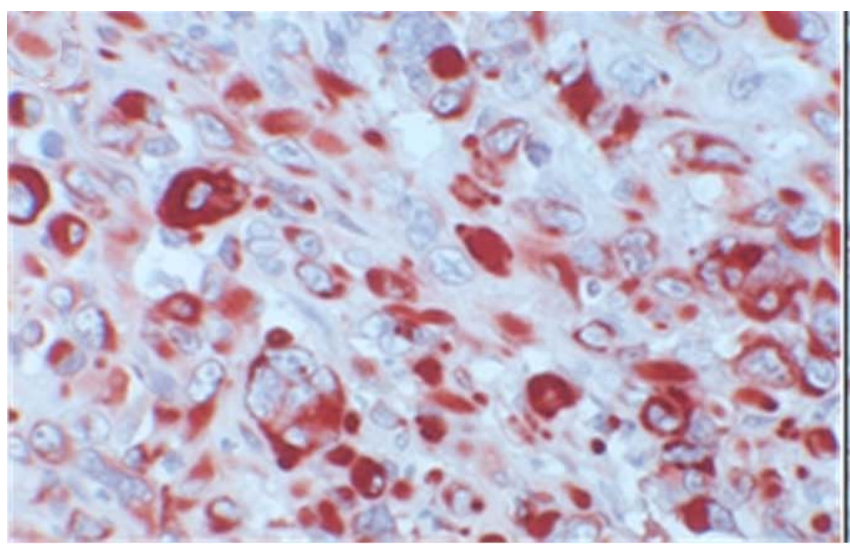

(c)

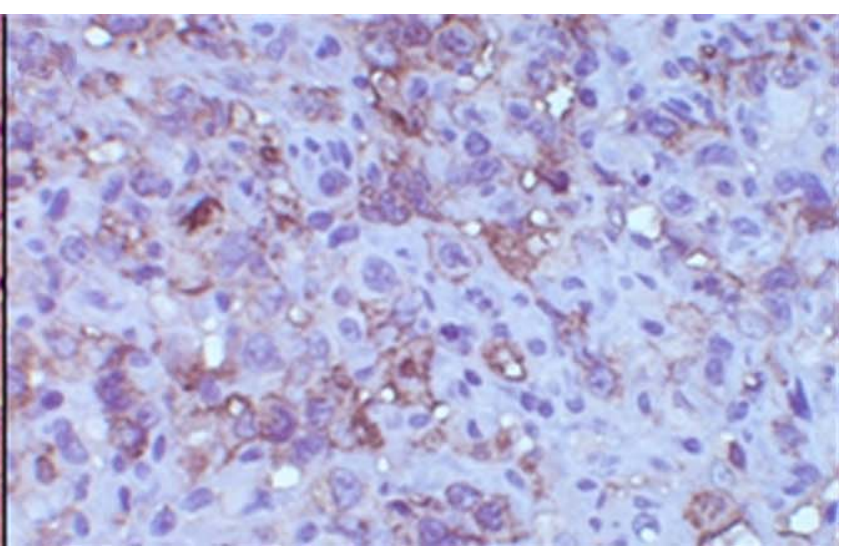

(b)

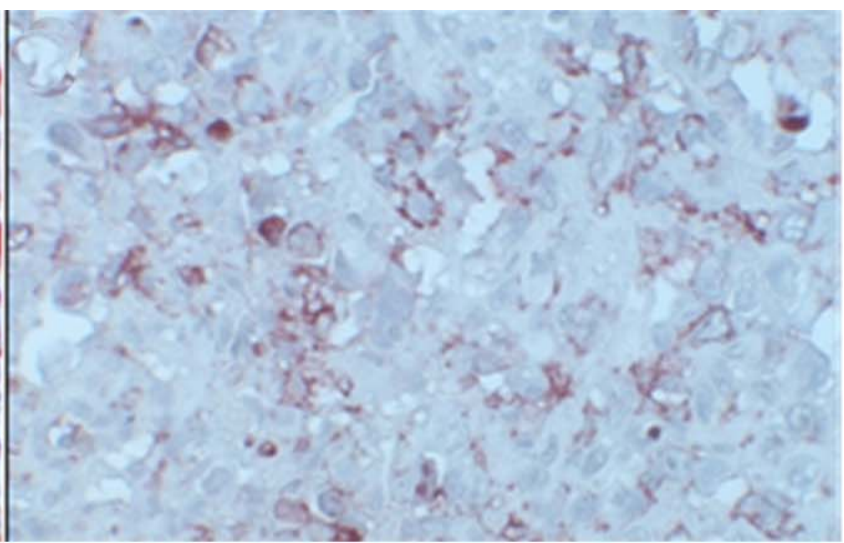

(d)

Figure 2. Pathologic findings. Tumor cells were arranged in a granuloma-like fashion around area of necrosis. The tumor cells showed plump and eosinophilic epithelioid appearance with nuclear atypical, vesicular chromatin, and numerous mitosis (X400) (a). On the immunohistochemical stain, the tumor cells revealed diffuse strong positive reaction in EMA (b) and cytokeratin (c). CD34 showed focal cytoplasmic positive reaction (d).

also showed accompanying bone destruction, not just demineralization, which is not a usual finding of epithelioid sarcoma (Figure 1).

Epithelioid sarcoma is a malignant tumor with an already poor prognosis, aggravated by the commonly delayed diagnosis and resulting inadequate treatment $[2,3]$. Therefore it is absolutely necessary to carry out immunohistochemical studies for the correct diagnosis. Typical for the epithelioid sarcoma is immunohistochemical positive for epithelial membrane antigen (EMA), cytokeratin, and vimentin but is negative for carcinoembryonic antigen and desmin [1-4]. Our case also revealed typical positive reaction in EMA, cytokeratin, and vimentin, although the initial pathologic diagnosis was missed.

Even with the initial treatment of choice, radical excision of the primary lesion, there is a high rate of local recurrence and lymphatic and pulmonary metastasis occurrence $[1,4,6,8]$. Many reports identify various factors about the prognosis; 1 ) the size and depth of the tumor, 2) the number of mitotic figures, and 3) the presence of hemorrhage, necrosis, and 4) vascular invasion [1,3,4,6, 8]. Three histology variables with vascular invasion, tumor size of more than $5 \mathrm{~cm}$, and more than $30 \%$ necrosis were reported as statistically significant predictors of a poor outcome [3]. According to these factors, the prognosis of our case was considered to be poor because of size greater than $5 \mathrm{~cm}$ and more than $30 \%$ necrosis on MR imaging and numerous mitosis and atypia in pathology. Unfortunately, this patient subsequently followed lymph node and rapid pulmonary metastasis in spite of en-bloc wide resection and adjuvant chemo- and radiotherapy.

\section{CONCLUSIONS}

Spinal epithelioid sarcoma is extremely rare and difficult to diagnose correctly. It also differs from other sarcoma subtypes because of its relative common recurrence and lymphovascular metastasis. When a spinal lesion has large necrotic paraspinal soft tissue mass with extension 
into the spinal canal in young adults, special immunohistochemical studies are recommended for determining the possibility of spinal epithelioid sarcoma.

\section{REFERENCES}

[1] Chase D.R., Enzinger F.M. (1985) Epithelioid sarcoma. Diagnosis, prognostic indicators, and treatment. The American Journal of Surgical Pathology, 9, 241-263. doi:10.1097/00000478-198504000-00001

[2] Bos G.D., Pritchard D.J., Reiman H.M., Dobyns J.H., Ilstrup D.M. and Landon G.C. (1988) Epithelioid sarcoma. An analysis of fifty-one cases. The Journal of Bone and Joint Surgery-American Volume, 70, 862-870.

[3] Halling A.C., Wollan P.C., Pritchard D.J., Vlasak R. and Nascimento A.G. (1996) Epithelioid sarcoma: a clinicopathologic review of 55 cases. Mayo Clinic Proceedings, 71, 636-642.doi:10.4065/71.7.636

[4] Kodet R., Smelhaus V., Newton W.A., Jr., et al. (1994) Epithelioid sarcoma in childhood: An immunohistochemical, electron microscopic, and clinicopathologic study of 11 cases under 15 years of age and review of the literature. Pediatric Pathology, 14, 433-451. doi:10.3109/15513819409024274

[5] Steinberg B.D., Gelberman R.H., Mankin H.J. and Rosenberg A.E. (1992) Epithelioid sarcoma in the upper extremity. The Journal of Bone and Joint Surgery-American Volume, 74, 28-35.

[6] Prat J., Woodruff J.M. and Marcove R.C. (1978) Epi- thelioid sarcoma: An analysis of 22 cases indicating the prognostic significance of vascular invasion and regional lymph node metastasis. Cancer, 41, 1472-1487. doi:10.1002/1097-0142(197804)41:4<1472::AID-CNCR 2820410436>3.0.CO;2-W

[7] Romero J.A., Kim E.E. and Moral I.S.(1994) MR characteristics of epithelioid sarcoma. Journal of Computer Assisted Tomography, 18, 929-931. doi:10.1097/00004728-199411000-00015

[8] Ross H.M., Lewis J.J., Woodruff J.M. and Brennan M.F. (1997) Epithelioid sarcoma: Clinical behavior and prognostic factors of survival. Annals of Surgical Oncology, 4, 491-495.doi:10.1007/BF02303673

[9] Steib J.P., Pierchon F., Farcy J.P., Lang G., Christmann D. and Gnassia J.P. (1996) Epithelioid sarcoma of the spine: A case report. Spine, 21, 634-638. doi:10.1097/00007632-199603010-00019

[10] Weisskopf M., Munker R., Hermanns-Sachweh B., Ohnsorge J.A. and Siebert C. (2006) Epithelioid sarcoma in the thoracic spine. European Spine Journal, 15, Suppl 5: 604-609.doi:10.1097/00007632-199603010-00019

[11] Yamato M., Nishimura G., Yamaguchi T., Tamai K. and Saotome K. (1997) Epithelioid sarcoma with unusual radiological findings. Skeletal Radiol, 26, 606-610. doi:10.1007/s002560050294

[12] Kawai A., Hasizume H., Sugihara S., Morimoto Y. and Inoue H. (2002) Treatment of bone and soft tissue sarcomas of the hand and wrist. International Orthopaedics, 26, 26-30.doi:10.1007/s002640100297 